\title{
Discriminating between reward-produced memories: Effects of differences in reward magnitude
}

\author{
E. J. CAPALDI, SUZAN ALPTEKIN, and KIMBERLY BIRMINGHAM \\ Purdue University, West Lafayette, Indiana
}

\begin{abstract}
The greater the dissimilarity between exteroceptive stimuli, the easier it is to discriminate between them. To determine whether a similar relationship holds for memories produced by reward events, rats in three runway investigations received trials in pairs, the number of food pellets $(0.045 \mathrm{~g})$ occurring on Trial 1 indicating whether reward or nonreward would occur on Trial 2. In each investigation, discriminative responding on Trial 2 was better the larger the difference in reward magnitude on Trial 1. This finding was obtained under a wide variety of conditions: for example, when the larger of two reward magnitudes on Trial 1 signaled nonreward on Trial 2 (Experiment 1, 10 vs. 2 pellets); when the smaller of two reward magnitudes on Trial 1 signaled nonreward on Trial 2 (Experiment 2, $10 \mathrm{vs}$. 2 pellets); and when the same magnitude of reward on Trial 1 signaled nonreward on Trial 2 (Experiment 3, either 5 pellets or 0 pellets). The findings obtained here indicate that the greater the dissimilarity between reward magnitudes, the greater the dissimilarity between the memories they produced and, thus, the easier it is to discriminate between them. It is suggested that the present results may provide a basis for understanding findings obtained in other instrumental learning investigations in which reward magnitude is varied.
\end{abstract}

A common procedure in instrumental discrimination learning investigations is to reward responses in the presence of some exteroceptive stimulus such as a visual or auditory stimulus (+ cue) and to nonreward responses in the presence of some other exteroceptive stimulus ( - cue). The present experiments were discrimination learning investigations of an unusual sort: The + and - cues were not exteroceptive cues but were interoceptive ones, the memories of prior reward events. The reasons for employing this unconventional form of discrimination learning may be briefly stated.

There is considerable evidence that reward-produced memories affect responding in a wide variety of instrumental learning investigations (Capaldi, 1994). Explaining the results of such investigations in terms of rewardproduced memories has typically involved the assumption that the memories have the same properties as exteroceptive stimuli. As one example, it has been assumed that the greater the dissimilarity between rewards in magnitude, the greater the dissimilarity in the memories they produce and, thus, the easier it is to discriminate between them (e.g., Capaldi, 1994). This assumption was tested in the three experiments reported here.

Reward-produced memories were differentially rewarded as follows. Rats received runway trials in pairs. Trials 1 and 2 of a pair were separated by a short temporal interval (about $15 \mathrm{sec}$ ). The pairs were separated by a long temporal interval (about $10 \mathrm{~min}$ ). Differential reward, such as 0 and 2 pellets $(0.045 \mathrm{~g})$, occurred on both Trial 1

Correspondence should be addressed to E. J. Capaldi, Department of Psychological Sciences, Purdue University, 1364 Psychology Bldg., West Lafayette, IN 47907-1364 (e-mail: julie@psych.purdue.edu). and Trial 2 of the pairs. Since the pairs occurred on a $50 \%$ irregular basis, the differential reward outcomes on Trial 1 could not be correctly predicted. However, the differential reward outcomes on Trial 2 were predictable. This was so because a given reward outcome on Trial 1 (e.g., 0 pellets) was always followed by the same reward outcome on Trial 2 (e.g., 2 pellets). Of major interest here was the development of discriminative responding on Trial 2 of the pairs.

Trial 2 behavior was expected to be regulated strongly, if not exclusively, by the memory of the Trial 1 reward and weakly, if at all, by any other reward-produced memories. It has been shown (e.g., Capaldi, Nawrocki, Miller, \& Verry, 1986) that when a long temporal interval elapses between trials (e.g., 10-20 min), any memory retrieved at that long interval has a strong effect on performance at that long interval and a weak effect, if any, on performance at a shorter interval (e.g., $15 \mathrm{sec}$ ). The same is true for memories retrieved at short temporal intervals. On the basis of those earlier findings, it follows that performance on Trial 2 of the pairs will be regulated primarily, if not exclusively, by the memory of the Trial 1 reward.

\section{EXPERIMENT 1}

Experiment 1 was designed to determine whether large reward ( $\mathrm{L}, 10$ pellets) is more easily discriminated from 0 pellets than is small reward ( $S, 2$ pellets). There were four groups employed in Experiment 1. Groups were designated in terms of the reward magnitude received on Trial 1 and Trial 2 of the pairs. For example, the designation $\mathrm{S} / \mathrm{L}$ means that small reward occurred on Trial 1 and that large reward occurred on Trial 2. Thus, Group S/L received Pairs 2-0 and $0-10$. Group S/S received Pairs 
$2-0$ and $0-2$. Group $\mathrm{L} / \mathrm{L}$ received Pairs $10-0$ and $0-10$. Group L/S received Pairs 10-0 and 0-2. In Experiment 1, the memory of 0 pellets stored on Trial 1 was always retrieved and rewarded on Trial 2. Thus, the memory of 0 pellets was a + cue. The memory of either 2 pellets or 10 pellets stored on Trial 1 was always retrieved and nonrewarded on Trial 2. Thus, the memories of 2 pellets and 10 pellets were - cues.

If 0 pellets is more easily discriminated from large reward than from small reward, then, on Trial 2 of the pairs, Groups L/L and L/S should show better discrimination responding than should Groups $\mathrm{S} / \mathrm{L}$ and $\mathrm{S} / \mathrm{S}$.

\section{Method}

Subjects. The subjects were 8 naive male albino rats (virus-free) about 80 days old when purchased from the Holtzman Co., Madison, WI.

Apparatus. The apparatus was a straight gray runway, $194.94 \mathrm{~cm}$ long, $8.8 \mathrm{~cm}$ wide, enclosed by $12.7-\mathrm{cm}$ sides covered by wire mesh on a hinged frame. The startbox was $23.3 \mathrm{~cm}$ long. The goalbox was $36.15 \mathrm{~cm}$ long. Raising the startbox door started a timer in a computer (IBM PC XT), which was stopped when the rat broke a photobeam located $158.13 \mathrm{~cm}$ beyond the startbox door. Pellets (45 mg, Noyes) were placed in a goal cup, which was $4.0 \mathrm{~cm}$ in diameter and $1.5 \mathrm{~cm}$ in depth, cut into a $6.5 \times 8.8 \times 4.5 \mathrm{~cm}$ block of wood. A brass door was lowered, confining the rats to the goalbox.

Pretraining. The rats were caged individually and given ad-lib food and water for 14 days after arriving at the laboratory. On Day 15 , pretraining began. On Day 1 of pretraining, food deprivation began, consisting of $16 \mathrm{~g}$ of Purina Lab Chow each day minus any amount eaten in the runway. On Days 1-10 of pretraining, the rats were handled. On Days 11-13 of pretraining, alley exploration occurred. On Days $11-13$, the rats were fed 6 pellets in the home cage and were allowed 3 min to explore the alley that had 6 pellets scattered about The startbox and goalbox doors were lowered and raised at about the 1.5-min mark in order to habituate the rats to noise.

Experimental training. After 13 days of pretraining, experimental training began and lasted 48 days. There were four groups, each consisting of 2 rats, in a $2 \times 2$ factorial design. Magnitude of reward on Trial 1 on $50 \%$ of the trials was either small (S) - that is, 2 pellets $(0.045 \mathrm{~g}$, Noyes) - or large (L) that is, 10 pellets $(0.045 \mathrm{~g}$, Noyes). On the other $50 \%$ of the trials, nonreinforcement $(0$ pellets) occurred on Trial 1. Trial 2 was likewise rewarded on half the trials with either 2 or 10 pellets and was nonrewarded on half the trials. Trials 1 and 2 occurred in pairs, trials of a pair being separated by about $15 \mathrm{sec}$ and pairs being separated by about $10 \mathrm{~min}$. Group S/L received Pairs 2-0 and 0-10: 2 pellets (or S) on Trial 1 always followed by 0 pellets on Trial 2 ( $2-0$ pair), and 0 pellets on Trial 1 always followed by 10 pellets (or $L)$ on Trial 2 (0-10 pair). Group S/S received Pairs $2-0$ and $0-2$. Group L/S received Pairs $10-0$ and $0-2$. Group $L / L$ received Pairs $10-0$ and $0-10$. Each pair was presented twice each day. Designate the $0-2$ or the $0-10$ pair as $A$ and the $2-0$ or the 10-0 pair as $B$. The order of presenting the pairs was ABBA (Day 1), BAAB (Day 2), BABA (Day 3), and $A B A B$ (Day 4). The pairs were presented in this order on each of the successive 4-day blocks. There were 48 days of experimental training. The 8 rats were taken into the experimental room in cages, which were placed in a rack. Each rat received its first pair before any rat received its next pair. This procedure produced an interval of about $10 \mathrm{~min}$ between pairs. On 0-pellet trials, the goal cup was false baited (i.e., pellets were placed in the goal cup as quietly as possible and were removed). On 0-pellet trials, the rat was removed from the goalbox after $15 \mathrm{sec}$. On reinforced trials, removal occurred immediately after the pellets were eaten. On all trials, the rat was placed in the start section. The start door was opened about
3 sec later. The order of running the rats was varied daily. The rat was allowed $30 \mathrm{sec}$ to reach the goalbox. If it did not, it was picked up and placed in the goalbox. After entering the goalbox or being placed there, the goalbox door was lowered. After the last rat was run, all rats were taken into the colony room and were fed the daily ration about $10 \mathrm{~min}$ later.

\section{Results}

A $2 \times 2$ analysis of variance (ANOVA) was applied separately to the running speeds on Trial 1 and on Trial 2. On Trial 1, only one significant difference of note was obtained: Speed of running on Trial 1 was greater, the larger the reinforcement magnitude on Trial $2[F(1,4)=9.91$, $p<.05]$. Mean speeds of running over all Trial 1 acquisition trials and on the last block of such trials were, respectively, 80.71 and $96.68 \mathrm{~cm} / \mathrm{sec}$ for the groups receiving small reward on Trial 2 . These means were 107.02 and $116.34 \mathrm{~cm} / \mathrm{sec}$, respectively, for the groups receiving large reward on Trial 2. On Trial 1, running speed increased over blocks $[F(11,44)=38.79, p<.001]$. However, no difference involving either groups or + versus - trials was significant on Trial 1 . Trial 1 data are not shown.

Figure 1 shows running speed on reinforced $(+)$ and nonreinforced $(-)$ trials on Trial 2 in blocks of 4 days. The left panel shows the running speeds for Groups S/L and $S / S$. The right panel shows the running speeds for Groups L/L and L/S. It is clear that Groups S/L and S/S had not developed the tendency to run slower on - trials than on + trials by the end of training. However, Groups $\mathrm{L} / \mathrm{L}$ and $\mathrm{L} / \mathrm{S}$ began to run faster on + trials than on - trials by about Block 4, a tendency that grew larger over subsequent blocks. A second major trend apparent in Figure 1 is this: Running was faster on Trial 2 when Trial 2 reinforcement was 10 pellets rather than 2 pellets. An ANOVA revealed the following on Trial 2: Running was faster on Trial 2, the smaller the reinforcement magnitude on Trial $1[F(1,4)=23.66, p<.01]$ and the greater the reinforcement magnitude on Trial $2[F(1,4)=27.33$, $p<.01]$, with the interaction of these variables being nonsignificant $(F<1)$.

Of greater concern is the finding that running speed was greater on + trials than on - trials $[F(1,4)=43.17$, $p<.01]$. The interaction of Trial 1 magnitude and + versus - trials was significant $[F(1,4)=51.56, p<.01]$, but the interaction of Trial 2 magnitude with + and - trials was not significant $[F(1,4)=5.52, p>.05]$.

\section{Discussion}

In Experiment 1, discriminative responding did not occur on Trial 1 and discriminative responding was not affected by the reward magnitude that occurred on Trial 2 of the pairs. On Trial 2, discriminative responding did occur. It was better in groups having the memory of 10 pellets as the - cue and 0 pellets as the + cue (Groups $\mathrm{L} / \mathrm{S}$ and $\mathrm{L} / \mathrm{L}$ ) than in groups having the memory of 2 pellets as the - cue and 0 pellets as the + cue (Groups S/L and $\mathrm{S} / \mathrm{S}$ ). Indeed, on Trial 2, Groups $\mathrm{S} / \mathrm{L}$ and $\mathrm{S} / \mathrm{S}$ did not respond discriminatively at all. The findings of Experi- 


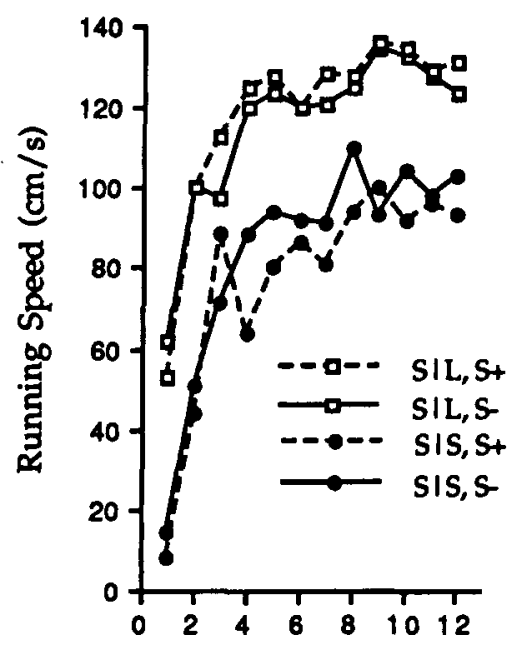

Blocks of 4 Days

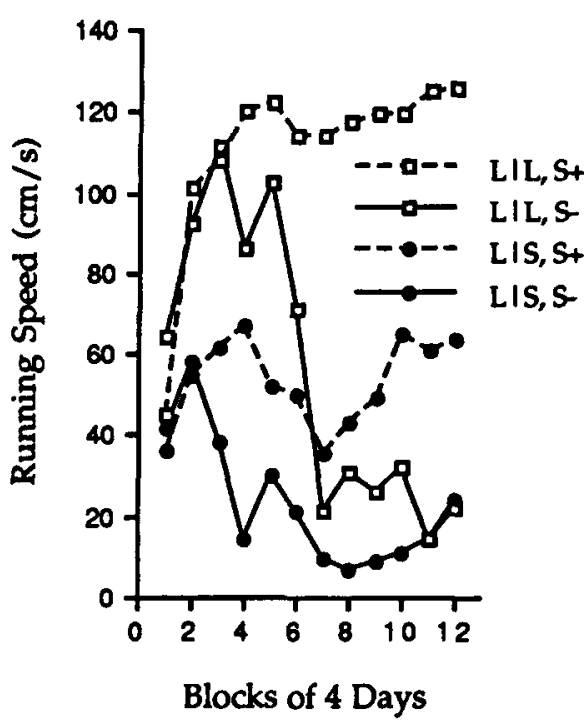

Figure 1. Running speeds on the Trial $2+$ and - trials in blocks of 4 days. Left panel: the speeds for Groups $S / L$ and $S / S$. Right panel: the speeds for Groups $L / L$ and $L / S$.

ment 1 are consistent with the view that the greater the difference between reward magnitudes, the easier it is to discriminate between the memories they produce.

An alternative interpretation of the results of Experiment 1 is available in terms of what might be called "contrast." According to that view, it may be easier to run slowly on a 0 -pellet trial that follows a large reward (e.g., 10 pellets) than on a 0 -pellet trial that follows a small reward (e.g., 2 pellets). The memory and contrast interpretations of Experiment 1 were tested in Experiment 2.

\section{EXPERIMENT 2}

In Experiment 2, two groups of rats were trained under conditions similar to those in Experiment 1. One group, Group 10-0, received Pairs 20-10 and $10-0$ and a single 2-pellet trial. The other group, Group 2-0, received Pairs $20-10$ and 2-0 and a single 10-pellet trial. Considering Trial 2, both groups had the same + cue, the memory of 20 pellets. The - cue was the memory of either 10 pellets (Group 10-0) or 2 pellets (Group 2-0). If, as the contrast view suggests, it is easier to run slowly on nonrewarded trials that follow large reward, as opposed to small reward, then Group 10-0 should show better discriminative responding on Trial 2 than should Group 2-0. However, if, as the memory view suggests, discriminative responding is more difficult the greater the similarity between the + and - cues, Group 10-0 should show poorer discriminative responding on Trial 2 than should Group $2-0$ because, of course, there is greater similarity between the memories of 20 pellets $(+)$ and 10 pellets $(-)$ than between the memories of 20 pellets $(+)$ and 2 pellets $(-)$. Groups $10-0$ and $2-0$ received the same overall magnitude of reward on Trial $1(20,10$, and 2 pellets) and on Trial 2 (10 and 0 pellets).

\section{Method}

Subjects. The subjects were 8 rats of the same description as those employed in Experiment 1.

Apparatus. The apparatus was the same as that employed in Experiment 1.

Pretraining. Pretraining was the same as that employed in Experiment 1.

Experimental training. After 13 days of preliminary training, experimental training began and lasted 50 days. There were two groups, each consisting of 4 rats. Unless otherwise specified, all procedures employed were the same as those in Experiment 1. Group 10-0 received Pairs $20-10$ and $10-0$ and a single 2-pellet trial. Group 2-0 received Pairs $20-10$ and 2-0 and a single 10-pellet trial. Designate the $20-10$ pair as A, the $10-0$ or the $2-0$ pair as $\mathrm{B}$, and the single 2-pellet or 10-pellet trial as $\mathrm{C}$. The order of presentation of these events was ABCCBA (Day 1), CABACB (Day 2), and BCABAC (Day 3). The events were presented in this order in each successive 3-day block.

\section{Results}

An ANOVA was applied separately to Trial 1 and Trial 2 speeds in blocks of 5 days. More data were available for analysis on Trial 1 ( 20 vs. 10 vs. 2 pellets) than on Trial 2 ( 10 vs. 0 pellets). Because of limitations in computer memory, two separate analyses were applied to the Trial 1 data, one over Blocks $1-5$ and another over Blocks 6-10. A single analysis was applied to the Trial 2 data. On Trial 1, differences associated with groups $(F<1)$ and with + versus - trials $(F<1)$ were not significant, and no differences involving either groups or + versus - trials were significant. On Blocks 1-5, running speed increased over blocks $[F(4,24)=49.68, p<.001]$. Mean running speed on Block 5 was $115.86 \mathrm{~cm} / \mathrm{sec}$ in Group 10-0, and it was $114.46 \mathrm{~cm} / \mathrm{sec}$ in Group 2-0. These speeds are very close to the asymptote reached by Block 10 . Over Blocks 6-10, running speeds stabilized, and differences due to blocks $(F<1)$ were not significant. 
Figure 2 shows running speed on reinforced $(+)$ and nonreinforced $(-)$ trials on Trial 2 for Group $10-0$ and Group 2-0 in blocks of 5 days. Group 10-0 failed to run faster on + trials than on - trials by the end of training. Group $2-0$, on the other hand, developed a strong tendency to run faster on + trials than on - trials starting by Block 3.

The difference due to groups was not significant $[F(1,6)$ $=2.13, p>.05]$. Importantly, the difference due to + versus - trials was significant $[F(1,6)=26.32, p<.01]$, as were both the + versus - trials $\times$ group interaction $[F(1,6)$ $=16.18, p<.01]$ and the + versus - trials $\times$ group $\times$ block interaction $[F(4,54)=6.11, p<.01]$.

\section{Discussion}

In Experiment 2, discriminative responding did not occur on Trial 1. In Experiment 2, Group 2-0 showed better discriminative responding on Trial 2 than did Group 10-0. These findings fail to support the contrast view outlined earlier; they are consistent with the proposition that the greater the dissimilarity in reward magnitude, the greater the difference in the memories they produce and, thus, the easier it is to discriminate between them.

Another alternative interpretation of the results obtained in Experiment 1 is rendered less likely on the basis of the results obtained in Experiment 2. In Experiment 1, the groups showing better discriminative responding on Trial 2 received the larger reward magnitude on Trial 1 (10 vs. 2 pellets). In Experiment 2, as indicated, Groups $10-0$ and $2-0$ received the same overall reward magnitude on Trial $1(10,2$, and 0 pellets). Thus, the results of Experiment 2 suggest that overall reward magnitude occurring on Trial 1 is not a major factor affecting discriminative responding on Trial 2, although the possibility that it may play some role cannot be dismissed on the basis of the findings reported here. Of course, in Experiment 2, Groups 10-0 and 2-0 received the same overall magnitude of reward on Trial 2 as well ( 20 and 0 pellets). Thus, the findings obtained in Experiment 2 appear

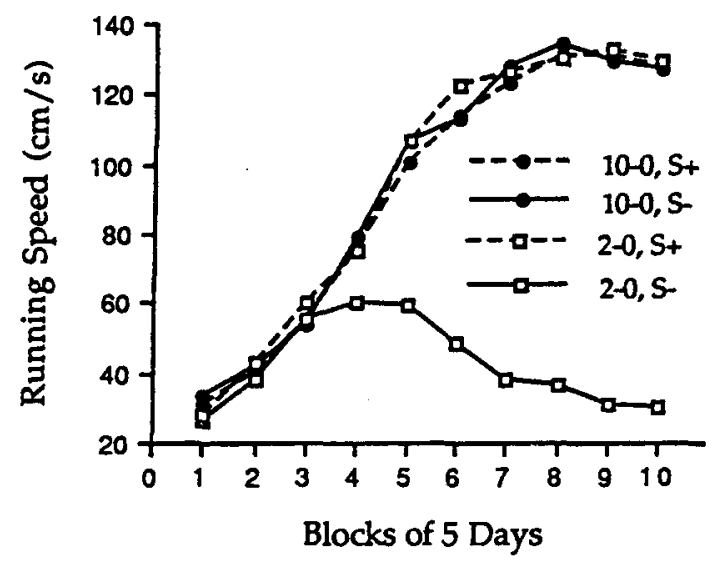

Figure 2. Running speeds on the Trial $2+$ and - trials in blocks of 5 days for Groups $10-0$ and $2-0$. to be independent of reward magnitude on both Trial 1 and Trial 2.

Experiment 3 was performed in order to test the present hypothesis under a wider and more severe set of conditions than that employed in Experiments 1 and 2.

\section{EXPERIMENT 3}

The results of Experiments 1 and 2 suggest that the greater the difference in the reward magnitudes employed as the + and - cues, the easier it is to discriminate between the memories they produce. This finding was obtained under the following conditions: The groups compared had the same + cue but had different - cues. Experiment 3 was designed to determine whether memories associated with larger differences in reward magnitude are more discriminable when the groups compared have different + cues and the same - cue.

In Experiment 3, Group 7-5 was trained employing Pairs 7-5 and 5-0 and a single 1-pellet trial. Group 1-5 was trained employing Pairs $1-5$ and 5-0 and a single 7 pellet trial. Following this training (original training), both groups were shifted to $0-0$ pairs. In the original training phase of Experiment 3, Group 1-5 should show better discriminative responding than should Group 7-5. This is because the memories of 1 pellet $(+)$ and 5 pellets $(-)$ are less similar than are the memories of 7 pellets $(+)$ and 5 pellets $(-)$. However, in the shift phase of Experiment 3 (0-0 pairs), the relation between the groups should be reversed, and Group 7-5 should run more slowly than should Group 1-5 following 0-pellet trials. This is because the memories of 1 pellet $(+)$ and 0 pellets $(-)$ are more similar than the memories of 7 pellets $(+)$ and 0 pellets $(-)$.

In Experiment 3, as in Experiment 2, the groups compared received the same overall magnitude of reward on Trial 1 and on Trial 2. In Experiment 3, on Trial 1, both groups received 1 pellet, 5 pellets, and 7 pellets (original training) and 0 pellets (shift). On Trial 2, both groups received 5 pellets and 0 pellets (original training) and 0 pellets (shift).

\section{Method}

Subjects. The subjects were 8 rats of the same description as those employed in Experiments 1 and 2.

Apparatus. The apparatus was the same as that employed in Experiments 1 and 2 .

Pretraining. Pretraining was the same as that employed in Experiments 1 and 2 .

Experimental training. After 13 days of preliminary training, experimental training began and lasted 50 days. There were two groups, each consisting of 4 rats. Unless otherwise specified, all procedures were the same as those in Experiment 1. Group 7-5 received Pairs 7-5 and 5-0 and a single 1-pellet trial. Group 1-5 received Pairs $1-5$ and 5-0 and a single 7-pellet trial. Designate the $7-5$ or the 1-5 pair as $A$, the 5-0 pair as B, and the single 1-pellet or 7-pellet trial as $C$. The order of presentation of these events was, as in Experiment 2, ABCCBA (Day 1), CABACB (Day 2), and BCABAC (Day 3$)$. The events were presented in this order in each successive 3 -day block. On Day 51, the rats were given four $0-0$ pairs. 


\section{Results}

An ANOVA was applied separately to Trial 1 and Trial 2 speeds in blocks of 5 days. As in Experiment 2, there were six trials for a Trial 1 analysis and four trials for a Trial 2 analysis. Because of limitations in computer memory, two separate analyses were applied to the Trial 1 data, one over Blocks 1-5 and another over Blocks 6-10. A single analysis was applied to the Trial 2 data. On Trial 1 , differences associated with groups $(F<1)$ and with + versus - trials $(F<1)$ were not significant, and no differences involving either groups or + versus - trials were significant. On Blocks $1-5$, running speed increased over blocks $[F(4,24)=51.38, p<.001]$. Mean running speed on Block 5 was $89.75 \mathrm{~cm} / \mathrm{sec}$ in Group 7-5, and it was $91.32 \mathrm{~cm} / \mathrm{sec}$ in Group 1-5. These speeds are very close to the asymptote reached by Block 10 . Over Blocks 6-10, running speeds stabilized, and differences due to blocks $(F<1)$ were nonsignificant.

Figure 3 shows running speed on reinforced $(+)$ and nonreinforced (-) trials of Trial 2 in blocks of 5 days for Groups $1-5$ and 7-5. Group 7-5 showed a mild tendency to run faster on + trials than on - trials by the last few blocks of trials. Group 1-5, on the other hand, showed a strong tendency to run faster on + trials than on - trials starting by about Block 5 .

The difference due to groups was not significant $[F(1,6)=1.19, p>.05]$. Importantly, the difference due to + versus - trials was significant $[F(1,6)=19.20, p<$ $.01]$, as were both the + versus - trials $\times$ group interaction $[F(1,6)=11.10, p<.05]$ and the + versus - trials $\times$ group $\times$ block interaction $[F(9,54)=4.15, p<.01]$.

On Trial 1 of the $0-0$ pairs, the difference due to groups was not significant $[F(1,6)=1.04, p>.05]$. On Trial 2 of the pairs, Group $1-5$ ran significantly faster than Group $7-5[F(1,6)=11.05, p<.05]$. The running speeds on each Trial 2 of the pairs are shown in Figure 4. As

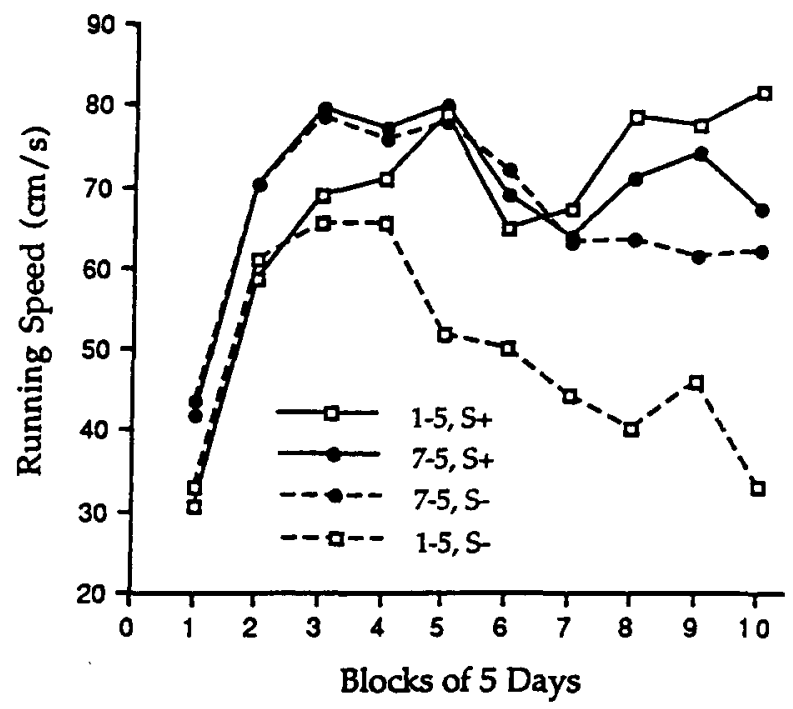

Figure 3. Running speeds on the Trial $2+$ and - trials in blocks of 5 days for Groups 7-5 and 1-5 for the same-phase portion of Experiment 3. may be seen, Group 7-5 ran slowly on the first Trial 2 of a $0-0$ pair. Group $1-5$, on the other hand, showed little tendency to slow down over the four $0-0$ pairs.

It may be of some interest to note the following. As may be seen by comparing Figures 1,2, and 3, running speeds on Trial 2 were lower in Experiment 3 than in Experiment 2 and were lower than running speeds of two groups in Experiment 1. It appears that running speed on Trial 2 was considerable only when the reinforcement magnitude on Trial 2 was 10 pellets. Reinforcement magnitudes of 2 pellets (Experiment 1 ) and 5 pellets (Experiment 3 ) on Trial 2 produced less than optimum speeds.

\section{Discussion}

In Experiment 3, it was found that a group having the memory of 7 pellets as the + cue showed poorer discriminative responding than did a group having the memory of 1 pellet as the + cue when the memory of 5 pellets was the - cue (original training). However, the group having the memory of 7 pellets as the + cue showed better discriminative responding than did the group having the memory of 1 pellet as the + cue when the memory of 0 pellets was the - cue (shift). Thus, in Experiment 3, as in Experiments 1 and 2, it was found that the greater the difference between two reward magnitudes, the easier it is to discriminate between the memories they produce. However, the experimental conditions employed in Experiment 3 differed in several respects from those employed in Experiments 1 and 2. In Experiment 3, discriminative responding was evaluated not only in an original training phase, as in Experiments 1 and 2, but in a shift phase as well. In Experiments 1 and 2, the groups compared had the same + cue (memory of 0 pellets in Experiment 1,20 pellets in Experiment 2) and different - cues (memories of 2 vs. 10 pellets). But, in Experiment 3, the groups compared had different + cues (memories of 1 vs. 7 pellets) and the same - cues (the memory of 5 pellets in original training and the memory of 0 pellets in shift).

\section{GENERAL DISCUSSION}

In the three experiments reported here, memories produced by reward events were differentially reinforced. It was hypothesized that the greater the difference in magnitude between reward events, the greater the differences in the memories they produce and, thus, the easier it should be to discriminate between them. This hypothesis was confirmed in the three experiments reported here under a wide variety of experimental conditions. It was confirmed when the groups compared had the same + cue and different - cues (Experiments 1 and 2) and when the groups compared had different + cues and the same - cue (Experiment 3). The hypothesis was confirmed both when the + and - trials were intermixed in an original training phase (Experiments 1,2, and 3) and when the trials were provided separately in a subsequent shift phase (Experiment 3 ). The hypothesis was confirmed both when the groups compared differed in the overall 


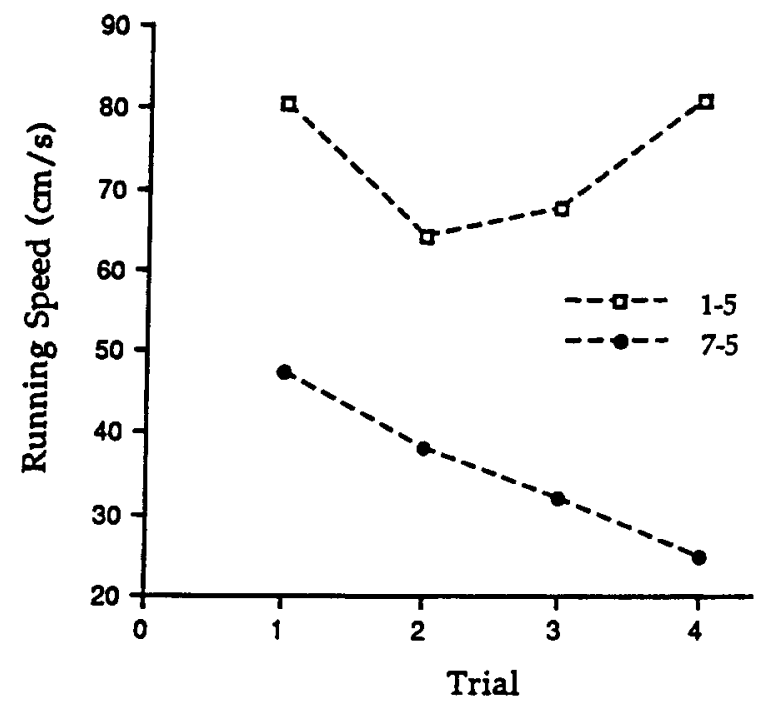

Figure 4. Running speeds on each of the Trial 2 trials of the $0-0$ pairs in Groups 7-5 and 1-5 for the separate-phase portion of Experiment 3.

magnitude of reward received on Trial 1 (Experiment 1) and when the groups were equated for overall magnitude of reward on Trial 1 and on Trial 2 (Experiments 2 and 3). The hypothesis was confirmed when the - reward magnitude was larger than the + reward magnitude (Experiment 1 ), when the - reward magnitude was smaller than the + reward magnitude (Experiment 2, the shift phase of Experiment 3), and when the - reward magnitude was both larger and smaller than the + reward magnitude (Groups 7-5 and 1-5, respectively, in the original training phase of Experiment 3). The hypothesis was confirmed when the magnitude of reward employed as the cue was larger in one group than in the other (Experiment 1), when it was smaller in one group than in the other (Experiment 2), and when it was the same in both groups (Experiment 3).

The findings obtained in the three experiments reported here may be interpreted to mean that the greater the difference between two reward magnitudes, the greater the differences in the memories they produce and, thus, the easier it is to discriminate between those memories. This finding may provide a basis for better understanding, at least in part, of findings obtained in other instrumental learning situations in which reward magnitude is varied. As one example, several laboratories have reported that resistance to extinction is greater following a small magnitude than following a large magnitude of consistent reinforcement (e.g., Hulse, 1958; Wagner, 1961). This extinction finding is consistent with the discrimination learning findings obtained here. To appreciate this, view extinction as a case of discrimination learning in which + cues occur in acquisition and - cues occur in extinction. Under a large magnitude of consistent reward, the memory of large reward is the + cue in acquisition; under a small magnitude of consistent reward, the memory of small reward is the + cue in acquisition. In extinction, the memories of nonreward is the - cue in both groups. It should be easier to discriminate between the relatively dissimilar memories of large reward and nonreward than between the relatively similar memories of small reward and nonreward. Thus, extinction should be more rapid, the greater the magnitude of consistent reward.

On the basis of the above analysis, it appears that findings obtained in extinction for different magnitudes of consistent reward are reasonably similar to those obtained in Experiment 1 in which better discriminative responding occurred for 10 pellets $(-)$ and 0 pellets $(+)$ than for 2 pellets $(-)$ and 0 pellets $(+)$. Extinction findings for different magnitudes of consistent reward seem to be highly similar to the shift findings obtained in Experiment 3 in which better discriminative responding occurred for 0 pellets $(-)$ and 7 pellets $(+)$ than for 0 pellets $(-)$ and 1 pellet $(+)$. Thus, in the shift phase of Experiment 3 , as in extinction following different magnitudes of consistent reward, it was easier to run slowly following a 0-pellet trial when the memory associated with the larger of two reward magnitudes had been rewarded. There are, of course, many differences between the three experiments reported here and extinction investigations. In Experiment 3 , for example, rats were nonrewarded prior to shift-something not true in shifts from consistent reward to extinction. In any event, other examples of instrumental learning phenomena that have been interpreted in terms of the degree of discriminability between reward-produced memories have been described elsewhere (e.g., Capaldi, 1994).

\section{REFERENCES}

CAPaldi, E. J. (1994). The sequential view: From rapidly fading stimulus traces to the organization of memory and the abstract concept of number. Psychonomic Bulletin \& Review, 1, 156-181.

Capaldi, E. J., Nawrocki, T. M., Miller, D. J., \& Verry, D. R. (1986) Time between events as a retrieval cue: Recall and the temporal similarity between the storage and retrieval intervals. Journal of Experimental Psychology: Animal Behavior Processes, 12, 258-269.

Hulse, S. H., JR. (1958). Amount and percentage of reinforcement and duration of goal confinement in conditioning and extinction. Journal of Experimental Psychology, 56, 48-57.

WAGNER, A. R. (1961). Effects of amount and percentage of reinforcement and number of acquisition trials on conditioning and extinction. Journal of Experimental Psychology, 62, 234-242.

(Manuscript received August 7, 1996; revision accepted for publication December 3, 1996.) 